Скопје, Македонија

\title{
ASSESSMENT OF THE RESCORLA-WAGNER MODEL WITH EXPERIMENTAL DATA
}

\author{
ROMAN GOLUBOVSKI, GJORGJI MARKOSKI, \\ GORDANA KITEVA-TRENCEVSKA, AND NATALIJA DOLNENEC-BANEVA
}

\begin{abstract}
Mathematical modeling is fundamental to the modern trends in the technological development, one of which is the Biomedical Engineering. This paper depicts an application in the field of EEG processing and analysis. Specifically, extraction (filtering) of event-related wave from within the overwhelming EEG potentials, and evaluation of a mathematical model of the cognitive process in humans supported by experimentally obtained data. CNV morphology is acquired through an experimental setup and subject's electroexpectogram (EXG) is derived. A suitable form of the Rescorla-Wagner model (basic for the classical conditioning theory) is solved as a system of differential equations, and one of the solutions is recognized to consistently match the EXG curve. At last, physiological explanation is given followed by a conclusion related to the experimentally supported mathematical model.
\end{abstract}

\section{INTRODUCTION}

Brain potentials are divided into spontaneous and event-related. The spontaneous result from the regular brain activity also known as the EEG potentials. The event-related potentials (ERP) result from external brain excitation (event) and can be divided into evoked and anticipatory. Evoked potentials appear after the excitation as a reflex of the brain. Anticipatory potentials appear before the corresponding event and represent an expectation of the same and usually a motor preparation process for it in the brain. The most prominent example of the expectation-related potential is the contingent negative variation $(\mathrm{CNV})$ potential. It is extracted from subject's EEG within the CNV experiment, originally proposed by Walter et al. [1]

2000 Mathematics Subject Classification. 92C50, 92C20, 91E10, 91E40.

Key words and phrases. Rescorla-Wagner model, EEG processing, electroexpectogram (EXG), contingent negative variation $(\mathrm{CNV})$. 
The CNV experiment is based on the CNV paradigm which applies two brain stimuli ( $S_{1}$ and $S_{2}$, usually audio) to the subject and with constant interstimulus interval (ISI). $S_{1}$ is a warning stimulus and $S_{2}$ is an imperative stimulus that the subject has to react on. The subject's reaction is applied to the experiment to prevent subject's concentration from lowering. The procedure is repeated tens of times, during which an ERP produced in the EEG trace between the stimuli shapes itself toward a specific CNV wave. The ERP after 10-20 trials can clearly show both components - the evoked (short) potential due to $S_{1}$ as well as the anticipatory (late, expectancy) potential together with the preparatory potential prior and due to $S_{2}$.

The DCNV (Dynamic CNV) experiment is an extension of the CNV experiment as defined above. The extension is actually a closed loop (biofeedback) which enables switching $S_{2} \mathrm{ON} / \mathrm{OFF}$ due to fulfilling certain conditions in the experiment's environment, thus forcing a cyclic process of building and degrading of the CNV wave. Subject is not informed about the nature of both stimuli, so the expectation of appearance (or absence) of $S_{2}$ during the experiment completely corresponds to the learning process. This allows modeling of the learning process.

The CNV wave (extracted ERP) can be qualified by one or combination of its parameters like amplitude, slope, etc. After the experiment, a statistical curve of the qualifying parameter (one of the mentioned) or combination is drawn across the trials. This statistical curve is denoted as the electroexpectogram (EXG) [2] and directly presents the subject's cognitive capabilities. Typical EXG curve is presented in figure 4. Clinical trials are expected to demonstrate distinctive differences in this statistics between different categories like healthy persons and patients with some kinds of neurological disturbances and different mental deteriorations.

As a conditioning paradigm, the DCNV completely corresponds to the classical conditioning paradigm, which is easily determined by comparing to its original example - Pavlov's experiment with dogs [3]. Actually, the only difference between the two experiments is the advantage of the DCNV paradigm that enables more evidence about the classical conditioning paradigm, since it gives evidence of the expectation process in the brain, not evident in Pavlov's experiment.

Attempts had been made to produce a mathematical model for the learning process, like the Rescorla-Wagner one, which is considered as fundamental in the theory of classical conditioning. This paper describes an experimental DCNV setup for producing a learning curve and tries to correlate it with the Rescorla-Wagner model's parameters. 


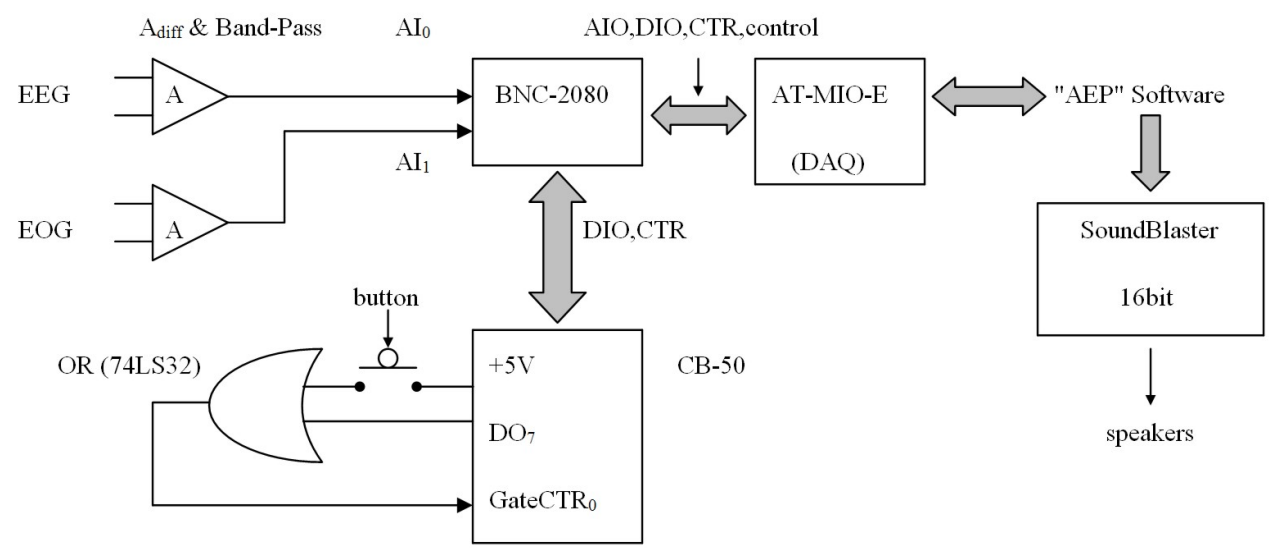

Figure 1: DCNV experiment the hardware configuration

\section{The Experiment Setup}

The experiment setup (given in figure 1) embraces the acquisition, signal processing and analysis of an EEG and EOG (electrooculographic) traces (latter used for validation of the EEG against artifacts) as well as reporting. The hardware used in the experiment is consisted of a precise Low-Pass amplifier for $\mu V$ ranges, a Data-Acquisition (DAQ) card, a sound card for stimuli application and a button with a TTL (Transistor-Transistor Logic) interface.

The system acquires two differential analogue channels, the EEG and the EOG. The excitation is audible, $S_{1}$ being a short $0.5 \mathrm{~s}$ ( 0.5 seconds) $1 \mathrm{kHz}$ warning beep and $S_{2}$ being a longer $(3.2 \mathrm{~s}) 2 \mathrm{kHz}$ imperative beep. It is essential that the subject is not aware neither of the nature nor of the number of the stimuli. The acquisition lasts for $7 \mathrm{~s}$ and is buffered and hardware timed. $S_{1}$ is issued in $t=1 \mathrm{~s}$ into the acquisition, $S_{2}$ is issued in $t=3 \mathrm{~s}$ if applied by the algorithm. During the experiment, the subject learns about the number, nature and order of the stimuli, thus demonstrating the process of learning by shaping the ERP wave toward the expected CNV. The subject has to react upon hearing $S_{2}$ by pressing the button and immediately interrupting it. This is a prevention from falling asleep and lowering of concentration. The number of trials in the experiment is set to maximum 100 successful (120 trials total). The gap between two successive trials varies from $12-15 \mathrm{~s}$ to avoid timing determinism. As mentioned in the introduction, the criterion for ERP being a CNV can be defined in several ways. It could be an ERP with amplitude at $S_{2}$ above predefined threshold, 
or an ERP with slope of its linearized interval between $S_{1}$ and $S_{2}$ above predefined threshold, or a combination of both. After three consecutive CNVs detected, $S_{2}$ is turned OFF and the subject learns to forget the imperative stimulus thus lowering the value of the CNV-qualifying parameter. After three consecutive NOT-CNVs (CNV not detected trials), $S_{2}$ is turned ON again, and so on. The EOG trace is used for automatic validation of the EEG trace against artifacts defined as voltage sequences longer and higher than preset thresholds. There is a second manual criterion applied, where the operator can reject current EEG if artifacts are recognised visually. Rejection of such trials is necessary since the process of extraction of the ERP uses a cumulative iterative FIR filter that averages the acquired signal by ansamble, so every artifact that passes it will influent the extracted ERP till the end of the experiment.

\section{The Software And Signal Processing}

The DCNV software [4] is engineered to support the experiment with the Institute of Physiology, Medical Faculty, University Ss. Cyril and Methodius - Skopje. Following are the descriptions of its interface and functionality. Main panel is given in figure 2 .

The main panel shows the acquired EEG signal in the current trial, the extracted CNV potential and its linearized model, as well as the required measurements and calculated values. The green (third) vertical marker on the CNV Morphology represents the reaction time of the subject. The yellow LED in the upper left corner of the REJECT button is ON for 3 s after the end of the current acquisition allowing the operator for that period of time to reject it if significant artifacts are noticed on the EEG strip. Patient Data is saved as a header in an ASCII data file. Options start and stop the experiment. Maximum 120 trials can be performed (average time duration limit of human patience as subject) but 100 successful are required. Measured values are the absolute offset $\left(r e f_{0}\right)$ and the reaction time $\left(R_{t}\right)$. Calculated values are the amplitudes of the CNV wave at $S_{1}$ and $S_{2}\left(\mathrm{~A}\left(S_{1}\right)\right.$ and $\left.\mathrm{A}\left(S_{2}\right)\right)$, having calculated the latencies of both stimuli, as well as the difference of the maximum and the minimum of the CNV signal in the ISI - the APP, the energy of the CNV wave in the ISI and the slope of the same calculated from the linearized model. Gain relates to the amplification, and c and d are parameters of the optimal cumulative filter for CNV extraction. The optimal filter is defined as follows:

$$
C N V_{i}=c \cdot C N V_{i-1}+d \cdot E E G_{i}
$$




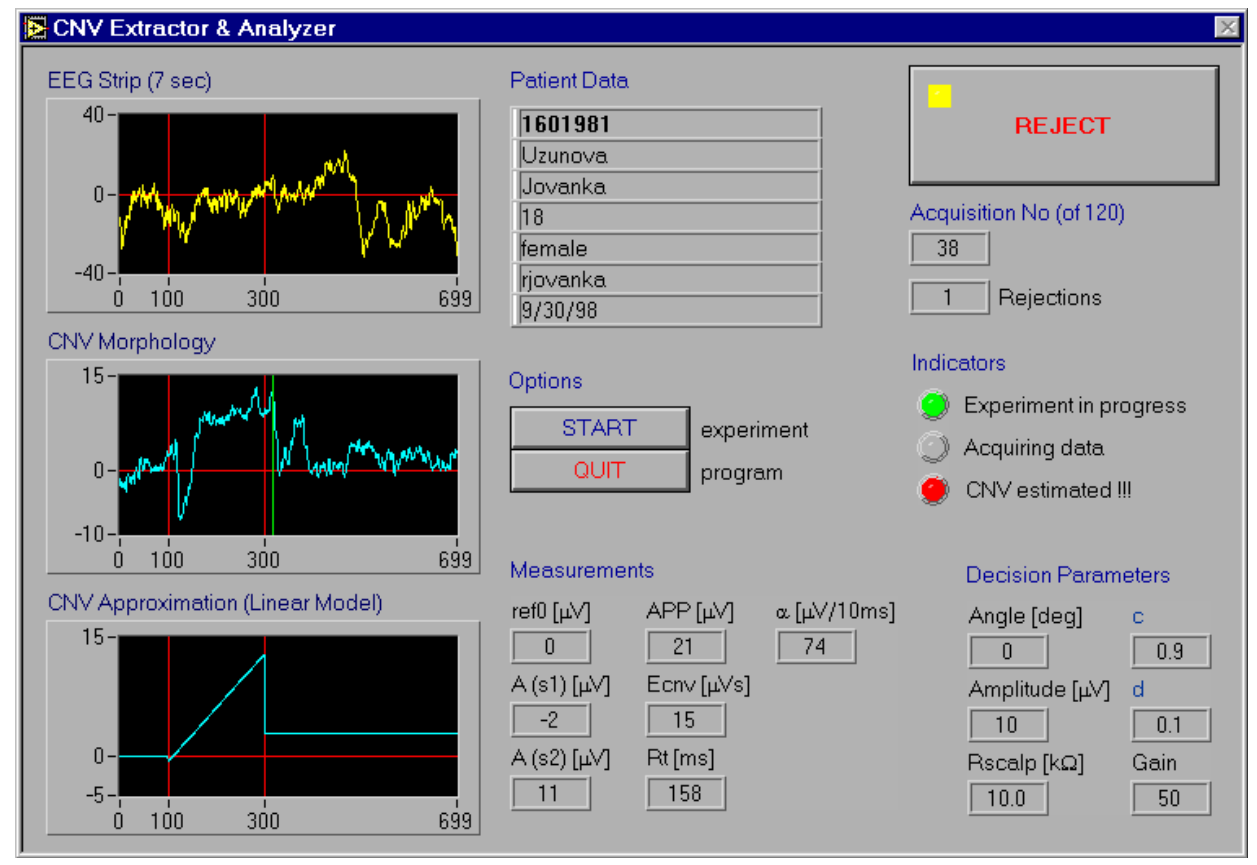

Figure 2: Main panel

or its explicite form:

$$
\begin{aligned}
& C N V_{n}=\sum_{i=1}^{n} c^{i-1} \cdot d \cdot E E G_{n-i+1} \\
& C N V_{0}=0
\end{aligned}
$$

The stability of the filter is obviously achieved by keeping $d<1$, and $d<c$ secures dominant influency of the current CNV accumulation in the current CNV extraction.

Complete report is retrieved from an ASCII file containing all acquired, processed and analyzed data. From the report figure 3 shows the complete EEG history across all valid (not rejected) trials, the complete CNV wave evolution during the experiment. Figure 4 provides the EXG curve as the crucial result of the experiment.

The application is completely hardware-synchronized. The acquisition is timed by the on-board clock of the acquisition card. The audio stimulation performed through the sound card is based on WAV strings prepared in the memory prior to the start of the experiment and triggered by the clock too. The reaction time is measured by the on-board counter, started by a digital output from the card issuing pulse at the same moment with the start of 

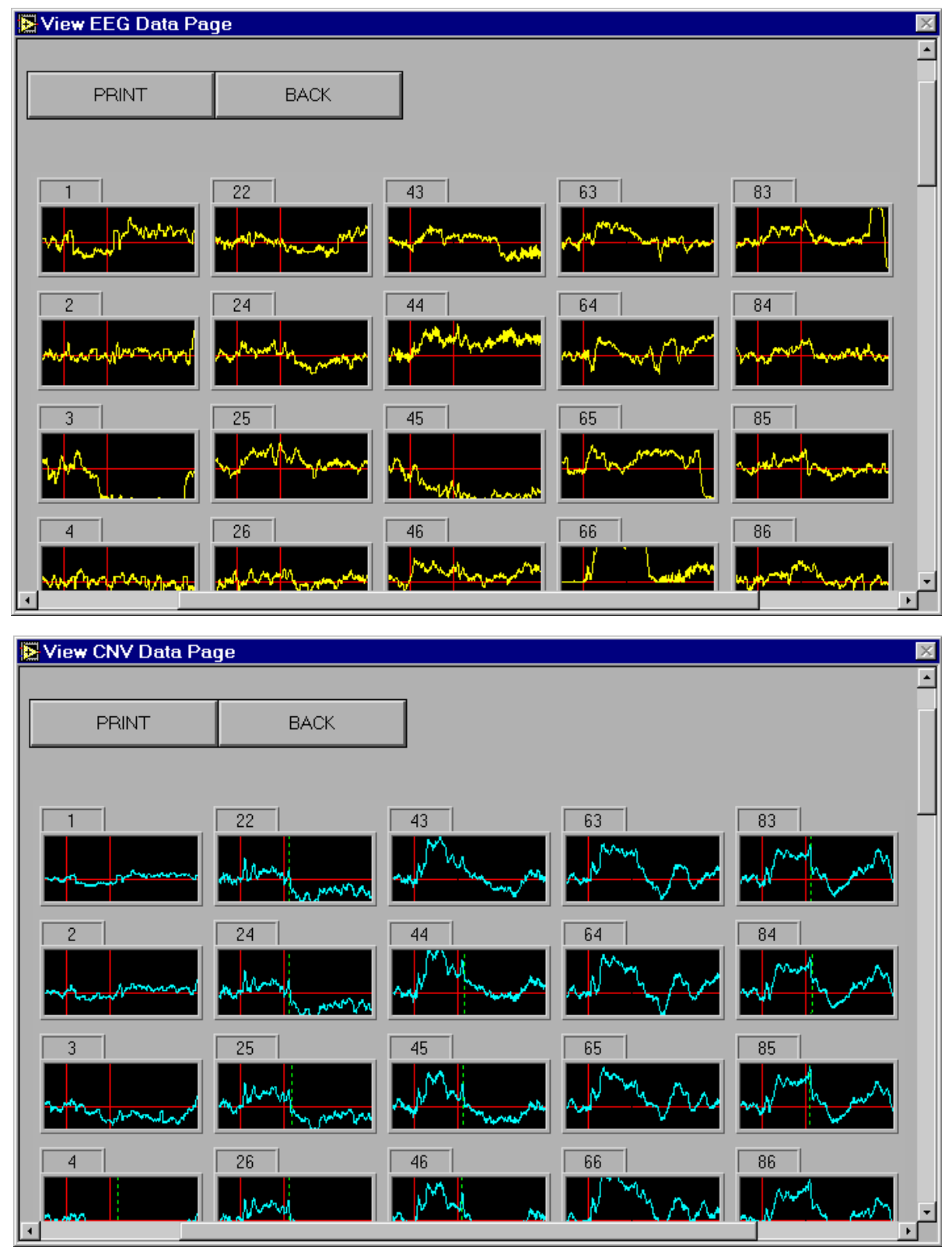

Figure 3: EEG history and CNV evolution

$S_{2}$ and stopped by the user press or the time-out pulse applied again by the same digital line. 


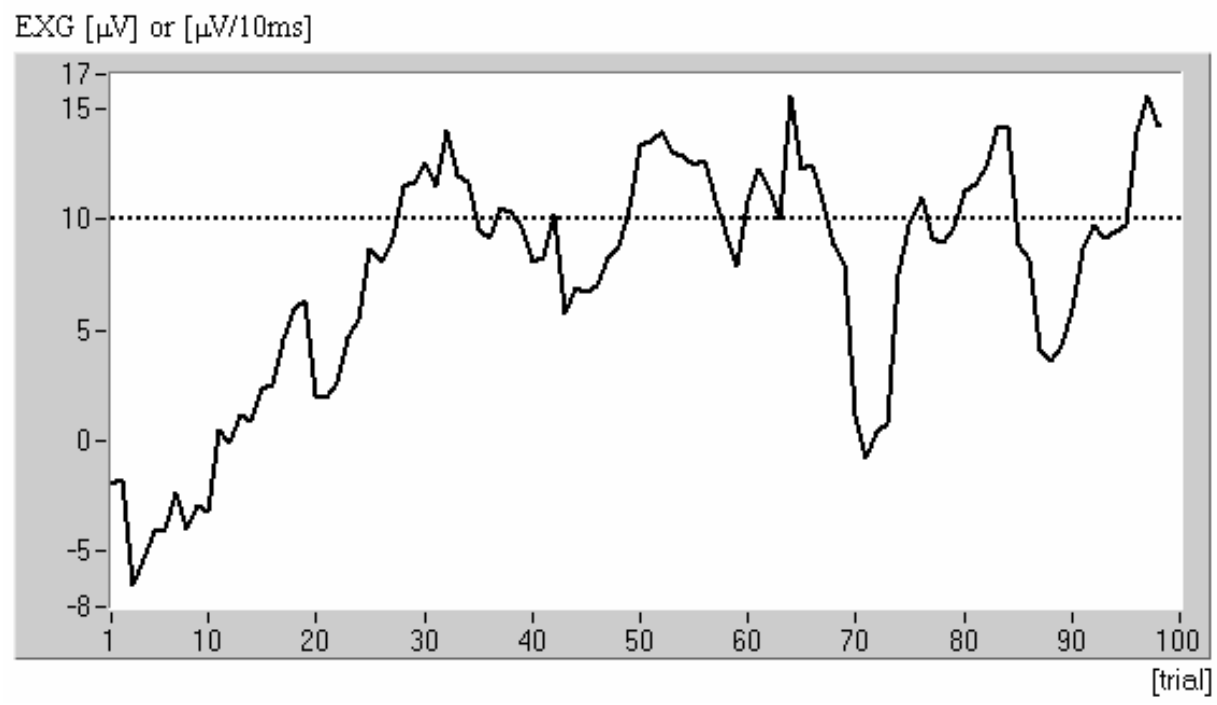

Figure 4: The EXG curve produced by the experiment

\section{The Rescorla-Wagner Model}

The Rescorla-Wagner model is the most influential theory of associative learning in the past 40 years. Based on precise basic assumptions [5] and details [6], a simplified model [7] is proposed as system of differential equations:

$$
\begin{aligned}
& x^{\prime}=a(100-x)+a(100-x-y) \\
& y^{\prime}=a(100-x-y)
\end{aligned}
$$

with initial conditions $x(0)=0, y(0)=0$, where $x=x(t), y=y(t)$. For visualization purpose the following modification is used instead:

$$
\begin{aligned}
& x^{\prime}=\frac{a}{b}(100-x)+\frac{a}{b}(100-x-y+b) \\
& y^{\prime}=\frac{a}{b}(100-x-y+b)
\end{aligned}
$$

with initial conditions $x(0)=0, y(0)=0$. The system (2) is solved with the computer software Mathematica, and presented in figure 5, with the following parameters: $a=0.65, b=10$. In this case $\lim _{t \rightarrow \infty} x(t)=100$ and $\lim _{t \rightarrow \infty} y(t)=10$.

The mathematical solutions are compared against experimental data of two subjects. The EXG curve is modified to be ploted in absolute values with respect to the CNV qualification threshold (as preset in the application experiment), by which the oscilatory component is removed and only the 
a)

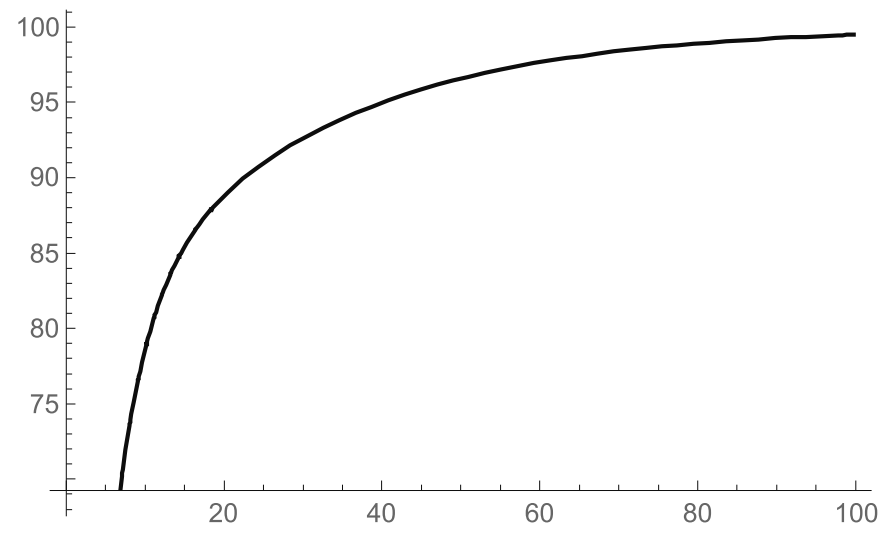

b)

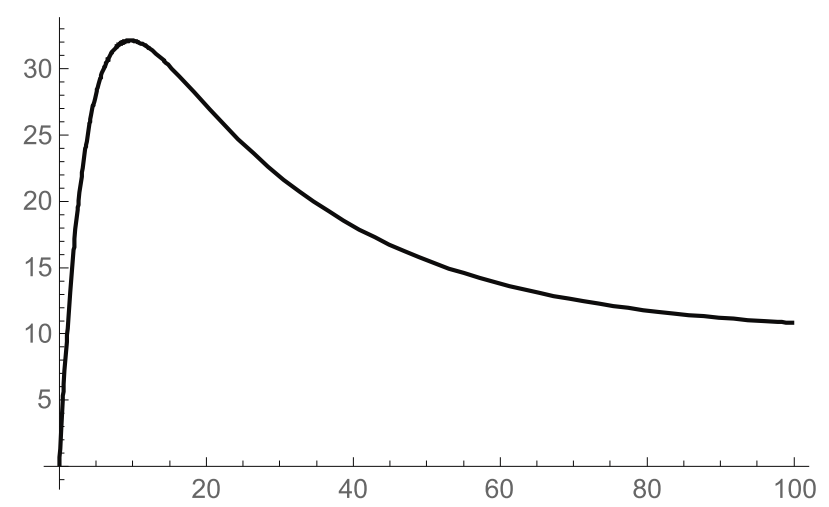

Figure 5: Solutions $x$ (presented in a)) and $y($ presented in $\mathrm{b}))$ for $a=0.25$ and $b=10$.

asymptotic convergence remains. The two experimentaly obtained curves are given in figure 6 .

As stated in the experiment setup above, the vector $E E G_{i}, i=1, \ldots, n$ is denoted as the $i$-th trial in the list of acquired sequences

$$
E E G_{i}=\left(E E G_{i}(j) \mid j=1, \ldots, 700\right),
$$

where $n$ is number of successful trials, and $\mathrm{j}$ is the sample index wihtin. Similarly, $C N V_{i}$ denotes the vectors of processed acquisitions after application of the filter (1) with parameters $d=0.1, c=0.9$ and $C N V_{0}=$ $(0 \mid j=1, \ldots, 700)$. As elaborated, for the purpose of removal of the oscilatory component (induced by the feedback in the exp eriment) absolute 


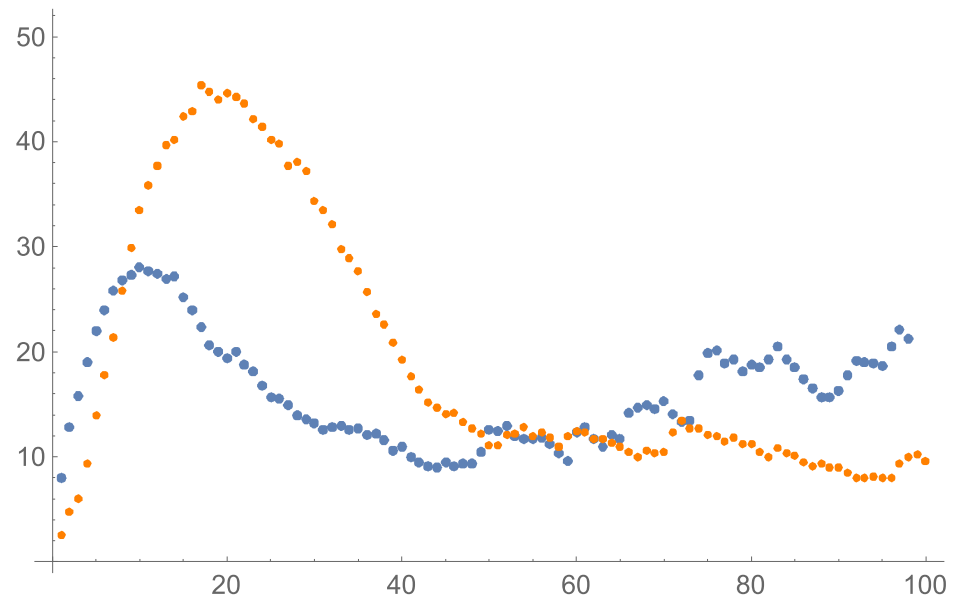

Figure 6: EXG curves for 2 patients

values of the CNV qualifications around the threshold are presented with

$$
\mid E E G_{i}-\text { threshold } \mid=\left(\mid E E G_{i}(j)-\text { threshold }|| j=1, \ldots, 700\right) .
$$

The modified EXG curves $\mid E X G_{i}-$ threshold $\mid$ are ploted in figure 6 . It is obvious that they both match one of the mathematical solutions for which a plausable physiological explanation can be given.

\section{CNV PARADIGM A WINDOW TO THE COGNITIVE FUNCTIONING}

Discovery of the CNV marks the birth of cognitive neuroscience. CNV is ERP, presented with a large, slow, negativity that grew in amplitude during the interval between $S_{1}$ and $S_{2}$, terminating abruptly with the subject's key-press response. CNV is entirely endogenous, it is not related to the characteristics of the stimuli themselves, but to the use that the subject makes of them (8). Hence CNV is task specific (9). Despite the debate about the underlined functional neuroanatomical basis of CNV $(10,11)$ it is linked to cognitive functioning and behavior performance and possibly several cognitive and sensory functions are involved during CNV generation like arousal, attention process, anticipation, preparration for reception and action, planning, working memory, learning process, habituation. It is shown that CNV differs in children and adults and these differences are explained with the maturation processes going on during developmental periods $(12,13)$. There are reports that CNV differs between patients with epilepsy and controls (14). Cognitive impairment is not rare in patients with epilepsy, due to different factors (the underlined pathology, side-effects 
from the pharmacotherapy, excessive cortical neuronal activity during ictal and interictal periods). There are reports that CNV may serve as a marker for disease progression and cognitive dysfunction in patients with multiple sclerosis (15). There are results showing differences in CNV amplitude between migraine patients and controls and these findings were used to follow the beta blockers treatment effects in migraine (16). CNV finds application in psychiatry, as CNV differs from healthy controls in patients with alcohol dependence syndrome, in patients with schizophrenia, with bibolar disorder and other psychopathologies $(17,18)$.

\section{Conclusion}

One of the goals of the DCNV experiment setup is to create subject's electroexpectogram. The EXG curve represents a cognitive wave obtained from the human brain showing the oscilatory change of the expectancy status in the human brain during the DCNV paradigm. The EXG is a manifestation of the expectation process and the learning process taking place in the human brain during the DCNV paradigm. The DCNV resarch software successfully performs the expected task and produces an excellent report containing all the required information usefull for the statistical research. Event-related potential negativity is interpreted as a representation of cortical excitability, facilitating the processing of sensory input, while positivity is a manifestation of neuronal inhibition. The modified EXG curve obtained by removing the oscillatory component during DCNV paradigm is related to the underlined cognitive processing and may be a represent of the physiologic CNS response to different tasks, different afferent stimuli and different consequent efferent outputs and the physiologic tendency for obtaining a basic homeostatic condition with balance in processes

of excitation and inhibition and a readiness for accepting new information to be processed in continuation.

\section{REFERENCES}

[1] G. Walter, R. Cooper, V. Aldridge, W. McCallum, Contingent negative variation: An electric sign of sensory motor association and expectancy in the human brain, Nature 203: 380-384, July 1964.

[2] L. Bozinovska, S. Bozinovski, G. Stojanov, Electroexpectogram: Experimental design and algorithms, Proc IEEE Biomedical Engineering Days, 58-60, Istanbul, August 1992.

[3] I. Pavlov, Conditioned Reflexes, Oxford University Press, 1927.

[4] R. Golubovski, DCNV Research Tool: Investigation of Anticipatory Brain Potentials, ESIT, Krete, Jun 1999. 
[5] R. Miller, R. Barnet, N. Grahame, Assessment of the Rescorla-Wagner Model, Psychological Bulletin 1995, Vol. 117, No. 3, 363-386.

[6] D. R. Shanks, Associative Versus Contingency Accounts of Category Learning: Reply to Melz, Cheng, Holyoak, and Waldmann (1993), Journal of Experimental Psychology: Learning. Memory, and Cognition, 1993. Vol. 19. No. 6. 1411-1423.

[7] M. Yamaguchi, New methods for solving the Rescorla-Wagner model, Behavior Research Methods, Instruments, \& Computers, 1999.31 (4). 684-688.

[8] S. A. Hackley, F. Valle-Incln, H. Masaki, K. Hebert Chapter 17 Stimulus-Preceding Negativity (SPN) and Attention to Rewards: Cognitive Electrophysiology of Attention, G.R. Mangun ed 2014 Elsevier 216-225.

[9] V. Sahai, OP. Tandon, Task related changes in contingent negative variation (CNV) response of endogenous evoked potentials, Indian J Physiol Pharmacol 2000; 44 (3):311-316.

[10] Y. Nagai, HD. Critchley, E. Featherstone, PB. Fenwick, MR. Trimble, RJ. Dolan, Brain activity relating to the contingent negative variation: an fMRI investigation, Neuroimage 2004; 21(4):1232-41.

[11] F. Fang, Y. Liu, Z. Shen, Lie detection with contingent negative variation, International Journal of Psychophysiology 2003. ;50:247-255.

[12] K. Anderson, The development of the contingent negative variation during an executive functioning/planning task in children, adolescents and adults, Thesis for the degree of master science University of Florida 2006.

[13] M. Siniatchkin, A. Jonas, H. Baki, A.van Baalen, W.D. Gerber, U. Stephani, Developmental changes of the contingent negative variation in migraine and healthy children, J Headache Pain (2010) 11:105113.

[14] M. E. Drake JR, S. J. Weate, S. A. Newell, Contingent negative variation in epilepsy, Seizure 1997; 6: 297-301.

[15] U. Uysal, F. Idiman, E. Idiman, S. Ozakbas, S. Karakas, J. Brucec Contingent Negative Variation Is Associated with Cognitive Dysfunction and Secondary Progressive Disease Course in Multiple Sclerosis, J Clin Neulol 2014; 10 (4): 296-303.

[16] I. Ahmed, Contingent Negative Variation in Migraine: Effect of Beta Blocker Therapy, Clin Electroencephalogr 1999; 30 (1):21-3.

[17] S. Sur, V. K. Sinha, Event-related potential: An overview, Ind. Psychiatry J 2009: 18 (1) $70-73$.

[18] Z. Li, W. Deng, X. Liu, Z. Zheng, M. Li, Y. Li, Y. Han, X. Ma, Q. Wang, X. Liu, T. Li, Contingent negative variation in patients with deficit schizophrenia or bipolar I disorder with psychotic features: measurement and correlation with clinical characteristics, Nord J of Psychiatry 2015 Apr;69(3):196-203. 
Faculty of Natural Sciences and Mathematics,

Ss. Cyril And Methodius University, Skopje, Macedonia

E-mail address: roman.golubovski@t.mk

InStitute OF MATHEMATiCs,

Faculty of Natural Sciences and Mathematics,

Ss. Cyril and Methodius University, Skopje, Macedonia

E-mail address: gorgim@pmf.ukim.mk

University Clinic of Neurology,

FACUlty OF Medicine,

Ss. Cyril and Methodius University, Skopje, Macedonia.

E-mail address: g.kiteva.trencevska@gmail.com

University Clinic of Neurology,

FaCulty of Medicine,

Ss. Cyril and Methodius University, Skopje, Macedonia.

E-mail address: natalijabaneva@yahoo.com 